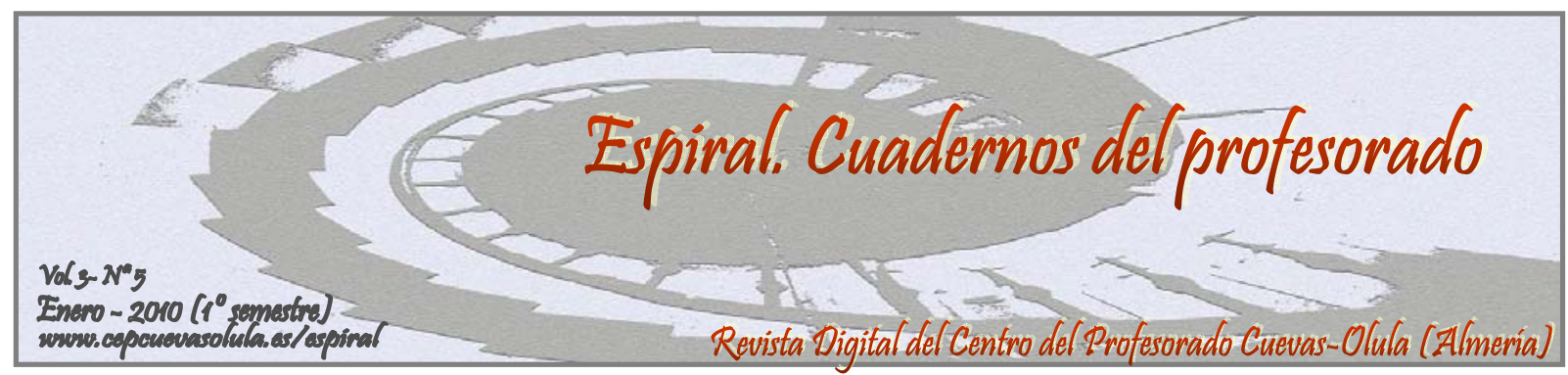

\title{
ACTIVIDADES FÍSICO-DEPORTIVAS QUE DEMANDAN LOS UNIVERSITARIOS
}

\section{PHYSICAL AND SPORTING ACTIVITIES THAT COLLEGE STUDENTS REQUIRE}

\author{
Dr. Manuel Gómez López, Dr. Francisco Ruiz Juan y Dra. Elena García Montes
}

\author{
Facultad de Ciencias del Deporte, Universidad de Murcia
}

RESUMEN: El presente trabajo aborda las características de la demanda de actividades físicas y deportivas de tiempo libre del alumnado universitario almeriense, para lo cual se ha seleccionado una muestra de 1.843 sujetos, a los que se les administró un cuestionario para evaluar las actividades en modalidad, periodo temporal, tipo de oferta y finalidad de las mismas. Durante la semana destacan las actividades físicas para el mantenimiento y la mejora de la salud, mientras que en fines de semana son los deportes individuales y colectivos los más practicados, sin perseguir un carácter competitivo. Las actividades en el medio natural están adquiriendo cada vez un papel más relevante durante los periodos vacacionales. Finalmente, la mayoría del alumnado prefiere realizar la práctica físico-deportiva por cuenta propia, de forma libre y autoorganizada.

Palabras clave: actividad físico-deportiva, tiempo libre, demanda, universitarios.

ABSTRACT: This paper is about the characteristics of physical-sport activities in leisure time of Almeria University students, for which it was chosen a sample of 1.843 students, who ware given a questionnaire to assess disciplines, period of time, kind of supply and goal activities. Along the week physical activities to maintain and improve their health, whereas in weekends, team and individual sports are more practiced, without a competitive character. Physical activities in nature environment are currently more important in holiday seasons. Finally, most of undergraduate prefer to do physical-sport activity by themselves, without timetable and organised by their own.

Key words: physical-sport activity, leisure time, demand, undergraduate

Gómez, M, Ruiz, F. \& García. M.E. (2010). Actividades físico-deportivas que demandan los universitarios. Espiral. Cuadernos del Profesorado [en línea], 3(5), 3-10. Disponible en: http://www.cepcuevasolula.es/espiral.

Fecha de recepción: 13/10/2009

Fecha de aceptación: 03/12/2009
Enviar correspondencia a: mgomezlop@um.es 


\section{1.- INTRODUCCIÓN.}

En la actualidad, tanto la actividad física como el deporte constituyen uno de los fenómenos sociales más importantes de la sociedad contemporánea, bien como práctica activa, bien como un espectáculo de masas, llegando ambos a formar parte esencial de la vida y cultura moderna (Consejo Superior de Deportes, 2000). Este deporte posmoderno se concibe como un sistema social abierto, al que se van incorporando nuevas prácticas y nuevas concepciones que van relativizando las correspondientes al deporte tradicional (Puig y Heinemann, 1991; 1992). Tampoco hay que olvidar, el progresivo incremento de la oferta de actividades físicas y deportivas de estos últimos años por parte de centros deportivos, gimnasios, patronatos municipales de deportes, clubes, etc..., que intentan dar respuesta a la interesante y amplia demanda de la población activa.

Tal y como afirmaba García Ferrando (2001), hoy día el problema para la población no es tanto el poder acceder a un uso y disfrute de actividades de ocio y tiempo libre, sino el seleccionar las actividades que mejor se adapten a sus gustos y posibilidades individuales. Se ha pasado de una perspectiva tradicional, donde el usuario tenía que adaptarse a la oferta, a la actual, donde es la oferta la que se amolda a los perfiles de sus demandantes. Esta amplia y diversa oferta de actividades físicas y deportivas tanto de empresas públicas como privadas, requiere una extensa y rigurosa planificación y gestión, con el fin de poder adaptarla a las características y necesidades de sus demandantes, así como propone Heinemann (1994), a los gustos y modas de un mercado cada vez más orientado por la concepción del deporte como objeto de consumo; de ahí que sea imprescindible el conocer cuáles son las actividades físicas y deportivas que prefieren y con las que les gustaría ocupar su tiempo libre a la población en cuestión.

La población a la que hacemos referencia en este estudio, es la universitaria, la cual se caracteriza por poseer una demanda plural y diversificada, con preferencias en sus comienzos por el deporte competitivo y organizado sobre todo en los varones, ya que conforme aumenta la edad, comienzan a decantarse hacia actividades menos competitivas, lo que pone de manifiesto un desfase entre la oferta deportiva tradicional universitaria $y$ las nuevas demandas de actividades físico-deportivas que poseen un mayor contenido recreativo (García Ferrando, 1990). Esta diversidad de inquietudes pueden y deben ser atendidas por el Servicio de Deportes de la Universidad, mediante una amplia y variada oferta físico-deportiva (Lizalde, 1993) que llegue a todo el alumnado (Salvador, 1994). Herrera (1996) resalta la existencia de una diversificada demanda universitaria, donde el deporte de recreación y el ocio se van imponiendo, unidos a cambios en la metodología de gestión de nuevas actividades desde los diferentes Servicios Deportivos.

En un trabajo realizado en las distintas universidades de las comunidades autónomas de Andalucía y Extremadura se concluyó que el alumnado universitario demanda actividades que no les obligue a seguir una pauta permanente y les prive de la libertad de utilizar su tiempo libre en su propia formación o en cualquier otra fórmula en la que ocupar su ocio (Tierra, 1995).

Estudios a nivel nacional como el realizado por García Ferrando $(1990 ; 1993)$ muestran que a los estudiantes universitarios les gustaría que desde sus universidades se promocionasen más cursos sobre el ámbito deportivo y recreativo, más actividades físicas, culturales y formativas, conferencias, cursos y campeonatos o ligas deportivas.

Por lo tanto, podemos decir que el deporte universitario se ha ido diversificando, dándose el caso de que la universidad plantee, en muchos casos, programas muy diferenciados, aunque no se llega a satisfacer la demanda de todos los usuarios a causa de la influencia de aspectos socio-políticos que los condicionan (Montiel y Sánchez, 1995).

Por todo lo anterior, en nuestra investigación, nos planteamos el conocer las características de la demanda de actividades físicas y deportivas de tiempo libre, del alumnado universitario almeriense, con el fin de disponer de datos objetivos que permitan tomar decisiones acertadas para la adecuación de la oferta físicodeportiva.

\section{2.- MATERIAL Y MÉTODO.}

La población objeto de estudio, está constituida por el alumnado universitario del curso académico 2001/02, quedando formada por 13.158 sujetos, de los cuales 9.602 alumnos son de primer ciclo (4.257 varones y 5.345 mujeres) y 3.556 alumnos de segundo ciclo (1.411 varones y 2.145 mujeres). Para realizar la selección de la 
Tabla 1. Demanda de actividades físico-deportivas en función de las variables sociodemográficas y académicas.

\begin{tabular}{llcc}
\hline & & Media & Demanda \\
\hline \multirow{2}{*}{ Edad } & De 18 y 20 años & $54.8 \%$ & $37.8 \%$ \\
& Más de 21 años & $45.2 \%$ & $62.2 \%$ \\
\hline \multirow{2}{*}{ Sexo } & Varón & $41.2 \%$ & $43 \%$ \\
& Mujer & $58.8 \%$ & $57 \%$ \\
\hline \multirow{2}{*}{ Estado civil } & Soltero & $92.3 \%$ & $94 \%$ \\
& Otro estado civil & $7.7 \%$ & $6 \%$ \\
\hline \multirow{2}{*}{ Facultades y } & Humanidades y Ciencias de la & $34.9 \%$ & $36.1 \%$ \\
& Educación & $22.1 \%$ & $15.1 \%$ \\
& Empresariales & $2.3 \%$ & $2.2 \%$ \\
& Escuela de Enfermería & $10.3 \%$ & $11 \%$ \\
& Ciencias Experimentales & $17.2 \%$ & $19.4 \%$ \\
& Escuela Politécnica Superior & $13.2 \%$ & $16.2 \%$ \\
\hline \multirow{2}{*}{ Turno de clase } & Derecho & $52.2 \%$ & $55.7 \%$ \\
& Mañana & $28.4 \%$ & $25.2 \%$ \\
\hline
\end{tabular}

muestra se utilizó un procedimiento de muestreo polietápico estratificado con afijación proporcional, asumiéndose un error muestral del $\pm 3 \%$ con un nivel de confianza del $95.5 \%$. La muestra representativa quedó establecida en 996 estudiantes de primer ciclo y 847 de segundo. El trabajo de campo se llevó a cabo en los meses de abril y mayo de 2002.

Atendiendo al objetivo del estudio se optó por una metodología cuantitativa, mediante encuesta, utilizando para ello como instrumento el cuestionario estandarizado "Hábitos deportivos y estilos de vida” (Ruiz Juan y García Montes, 2005), el cual fue autoadministrado mediante aplicación masiva por aulas y en presencia de un aplicador.

La información recogida se analizó con el programa estadístico SPSS para Windows
(V11.0), que posibilitó la puesta en práctica de las técnicas estadísticas de análisis descriptivo.

\section{3.- RESULTADOS.}

Al alumnado universitario se le planteó la siguiente cuestión: "Quisiéramos saber si te gustaría hacer o no actividad física y deportiva en tu tiempo libre. En caso afirmativo, ¿qué modalidades de actividades físicas y/o deportivas te gustaría realizar, en qué tipo de oferta y con qué finalidad?”. Se les pidió que indicasen, por orden de preferencia, las modalidades de actividades físico-deportivas que les gustaría hacer en su tiempo libre (incluyendo tanto aquellas que ya practicasen y quisiesen seguir practicando, como todas aquellas nuevas que deseen practicar), la frecuencia, el periodo temporal (días laborables, fines de semana y periodos vacacionales), el tipo de oferta (por tu

Tabla 2. Características de las actividades físico-deportivas en función del periodo temporal

\begin{tabular}{|c|c|c|c|c|}
\hline & & $\begin{array}{c}\text { Días } \\
\text { Laborables }\end{array}$ & $\begin{array}{l}\text { Fines de } \\
\text { Semana }\end{array}$ & $\begin{array}{c}\text { Periodos } \\
\text { Vacacionales }\end{array}$ \\
\hline \multirow[t]{4}{*}{ Modalidades } & Deportes Colectivos & $30.1 \%$ & $29.3 \%$ & $25.5 \%$ \\
\hline & Deportes Individuales & $28.4 \%$ & $29.2 \%$ & $32.2 \%$ \\
\hline & Act. Físico-deportivas & $35.5 \%$ & $15.2 \%$ & $14.6 \%$ \\
\hline & Act. físico-deportivas en el medio natural & $6.1 \%$ & $26.4 \%$ & $27.6 \%$ \\
\hline \multirow{4}{*}{ Tipo de oferta } & Por tu cuenta & $37.4 \%$ & $49.9 \%$ & $54.3 \%$ \\
\hline & Privada & $15.3 \%$ & $9.5 \%$ & $8.1 \%$ \\
\hline & Pública & $28.1 \%$ & $24.5 \%$ & $27.3 \%$ \\
\hline & Universidad de Almería & $19.2 \%$ & $16.1 \%$ & $10.3 \%$ \\
\hline \multirow{3}{*}{ Finalidad } & Competición federada & $8.8 \%$ & $7.2 \%$ & $4.8 \%$ \\
\hline & Ligas o trofeos no federados & $9.4 \%$ & $9.6 \%$ & $7.5 \%$ \\
\hline & Sin carácter competitivo & $81.8 \%$ & $82.8 \%$ & $87.7 \%$ \\
\hline
\end{tabular}


cuenta, privada, pública y universitaria) y la finalidad de dichas prácticas (competición federada, ligas ó trofeos no federados y sin carácter competitivo).

Los resultados obtenidos muestran que la tasa de universitarios demandantes de actividades físico-deportivas es superior al sesenta por ciento (64.1\%). Una vez analizadas las variables sociodemográficas (sexo, edad y estado civil) y de vida académica (Facultad/Escuela Universitaria y turno de clase) se aprecia que los alumnos que parecen tener más claro que quieren realizar actividades físico-deportivas en el futuro y por lo tanto más la demandan con respecto a la media, son los mayores de 21 años, el colectivo de varones y los solteros (tabla 1).

En cuanto a las variables académicas, apreciamos que tanto en la Facultad de Derecho, como en la de Humanidades y Ciencias de la Educación y en la de Ciencias Experimentales, se hallan las mayores concentraciones de peticiones de actividades físico-deportivas, estando los porcentajes, levemente por encima de la media, mientras que en otras como la Escuela de Enfermería se sigue la tendencia central (tabla 1).

El turno de clase, refleja que son los alumnos matriculados en el turno de mañana, los que más demandan este tipo de actividades, siguiendo la tendencia general de la población aquellos que cursan estudios en el turno de mañana y tarde, ya que sus porcentajes varían muy levemente con respecto a los encontrados en la descriptiva general (tabla 1).

A continuación vamos a ir mostrando cuales son las modalidades y actividades físicodeportivas con mayores tasas de demanda entre el alumnado universitario comenzando de manera general para finalizar con un análisis en los diferentes periodos temporales, junto al tipo de oferta y finalidad. De manera global, la demanda de modalidades físico-deportivas queda distribuida de forma bastante equilibrada, ya que el treinta por ciento de los alumnos solicitan deportes individuales (30.2\%), tan solo algo más de dos unidades porcentuales que los que piden deportes colectivos (27.9\%). Finalmente, encontramos que el resto de porcentaje se distribuye casi de manera equitativa entre los demandantes de actividades físico-deportivas en el medio natural y los que piden actividades físico-deportivas con fines saludables, es decir, aquellas que persiguen el mantenimiento o mejora del estado de salud (20.6\% y $21.3 \%$ respectivamente) (figura 1 ).

Más concretamente, observamos que las modalidades más solicitadas por los universitarios, dentro de los deportes colectivos son el fútbol (8.2\%), el baloncesto (6.3\%), el voleibol (5.8\%) y el fútbol-sala (3,5\%). En los deportes individuales destaca la natación (12.8\%), el tenis $(7,5 \%)$ y el ciclismo $(3,5 \%)$, en las actividades físico-deportivas el aeróbic (7.9\%), la carrera continua (3,9\%) y la musculación $(2,8 \%)$ y dentro de las actividades en el medio natural el senderismo (5.5\%). En el resto de las modalidades se han obtenido porcentajes inferiores al dos por ciento.

En cuanto a las actividades más demandadas por los universitarios los días laborables, los resultados muestran que son las actividades físico-deportivas (35.5\%) las más solicitadas, seguidas por los deportes colectivos (30.1\%) e individuales (28.4\%), a distancia de las que se desarrollan en el medio natural (6.1\%) (tabla 2). Con respecto a las modalidades, hay que resaltar el aeróbic (14.4\%), natación (10.9\%), fútbol (8\%), tenis (6.2\%), baloncesto (5.9\%), voleibol (5.2\%) y musculación (5.1\%).
Figura 1. Demanda de actividades físico-deportivas en función de la modalidad deportiva.

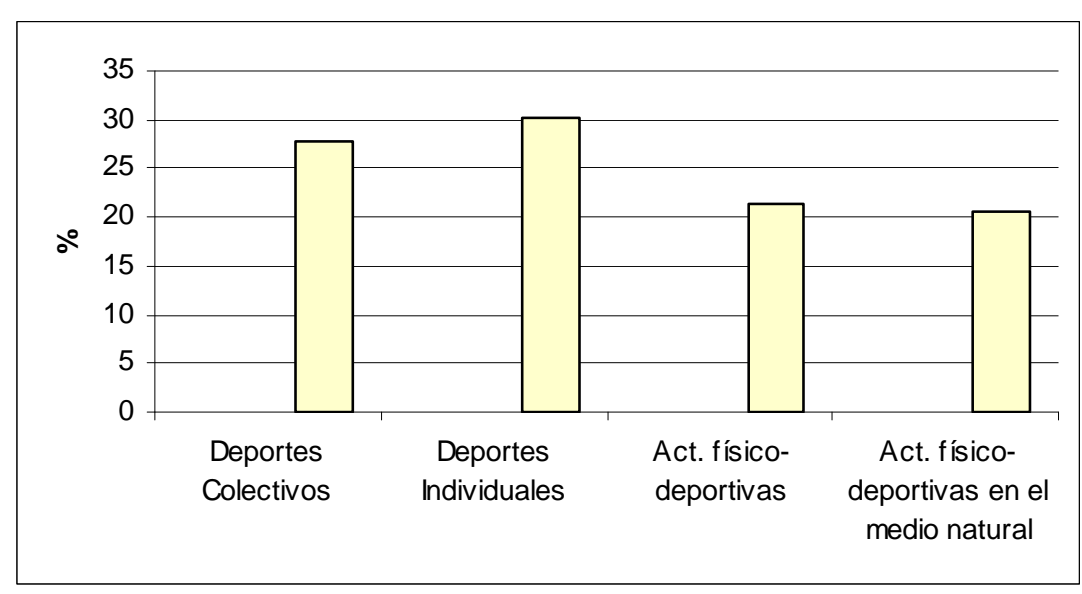


En cuanto al tipo de oferta, apreciamos que más de un tercio del alumnado solicita realizar las actividades físico-deportivas por su cuenta (37.4\%), opción que supera en casi diez puntos a la oferta pública (28.1\%). En tercer y cuarto lugar, quedarían las ofertas de la propia Universidad y la privada $(19.2 \%$ y $15.3 \%$ respectivamente). Más del ochenta por ciento de los estudiantes, demandan este tipo de actividades con una finalidad no competitiva (81.7\%), quedando distribuido el resto casi por igual entre los que desean ligas ó trofeos no federados (9.7\%) y los que quieren competiciones federadas (8.6\%) (tabla 2).

En los fines de semana, la demanda físicodeportiva se distribuye de manera casi equitativa entre los que desean deportes colectivos e individuales (29.3\% y $29.2 \%$ respectivamente). En tercer lugar $\mathrm{y}$ a tan solo tres puntos, encontramos aquellos que solicitan actividades físico-deportivas en el medio natural (26.4\%), para finalizar con el resto que preferiría practicar actividades físico-deportivas con fines de salud (15.2\%) (tabla 2). Los resultados indican que en este periodo temporal, a la mayoría del alumnado les encantaría realizar sobre todo senderismo (10.4\%), tenis (9.8\%), fútbol (8.8\%), natación (7.8\%), baloncesto (7.1\%) y voleibol (6.1\%).

Casi la mitad del alumnado preferiría realizar estas actividades por su cuenta (49.9\%), seguida a distancia de la oferta pública (24.5\%), la propia de la Universidad (16.1\%) y la privada (9.5\%). En cuanto a la finalidad con la que les gustaría practicar, apreciamos que sin duda alguna, es con un carácter no competitivo (82.8\%), a muchísima distancia de la demanda de competiciones federadas $y$ no federadas $\left(\begin{array}{llll}7.2 \% & y & 9.6 \%\end{array}\right.$ respectivamente) (tabla 2 ).

Finalmente, durante los periodos vacacionales de Navidad, Semana Santa y verano, la mayoría del alumnado preferiría ocupar su tiempo libre con la práctica de deportes individuales (32.2\%). En segundo y tercer lugar, estaría las actividades físico-deportivas en el medio natural y los deportes colectivos $(27.6 \%$ y $25.5 \%$ respectivamente). Las modalidades más solicitadas son la natación (16.6\%), tenis (7.1\%), fútbol y senderismo $\quad(6.6 \%$ y $6.5 \%$ respectivamente) y baloncesto y voleibol (5.1\% y $5 \%$ respectivamente). A la mayoría del alumnado le gustaría practicar por su cuenta (54.3\%), seguidos de aquellos que prefieren la oferta pública $(27.3 \%)$, la propia de la Universidad (10.3\%) y la privada (8.1\%). En cuanto al carácter de la práctica, los resultados muestran que casi el noventa por ciento de ellos desearían hacerlo sin ningún carácter competitivo (87.7\%), distribuyéndose el resto del porcentaje entre los que preferirían ligas ó trofeos no federados (7.5\%) y los que solicitan competiciones federadas (4.8\%) (tabla 2).

\section{4.- DISCUSIÓN.}

Es interesante destacar que los resultados muestran claras tendencias a realizar determinadas actividades en cada uno de los periodos temporales analizados, así mientras que durante la semana, los estudiantes desean sobre todo realizar actividades físico-deportivas cuyo objetivo es el mantenimiento y mejora de su estado de salud, en los fines de semana son los deportes tanto colectivos como individuales los más solicitados, siendo esta última modalidad deportiva la más demandada también en los periodos vacacionales. Hay que resaltar también la importancia que va teniendo la práctica de actividades físico-deportivas en el medio natural tanto en fines de semana como en los distintos periodos vacacionales analizados.

En este caso, al igual que en estudios anteriores referidos a la demanda de actividades físico-deportivas de tiempo libre en la Universidad de Almería, como los de Hernández Rodríguez (2001), Ruiz Juan (2001), Hernández Rodríguez, García Montes y Oña Sicilia (2002) y Ruiz Juan y García Montes (2002; 2004), y en la línea de los resultados hallados entre los universitarios de Álava por García de la Torre y Antón (1990), los deportes individuales junto a los colectivos son los más demandados por la población investigada, destacando la natación y el tenis entre los primeros y el fútbol, voleibol y baloncesto entre los segundos. Igualmente, y en el caso de la natación, los resultados son similares a los encontrados en otros estudios donde esta actividad no reglada se sitúa en primera posición de la demanda físico-deportiva, llegando a considerar la piscina cubierta como el principal soporte para la práctica de actividades acuáticas y una de las instalaciones deportivas más necesarias (Cañellas y Rovira, 1995; Rodríguez et al., 2006).

Estos estudios referidos al alumnado almeriense, al igual que el presente, muestran la importancia de las actividades físico-deportivas más relacionadas con la salud, como el aeróbic y la gimnasia de mantenimiento, actividades muy practicadas a estas edades debido a la 
preocupación por la estética y por la imagen personal, aunque la mayoría de ellas sean practicadas como veremos a continuación, en instalaciones ajenas a la propia Universidad.

La diversificación de la demanda se hace patente y las actividades de recreación y de ocio se van imponiendo, de manera notable, al igual que en la sociedad, entre el alumnado, especialmente el universitario, obligando a modificar la oferta, en función de estas nuevas tendencias. Tendencia similar a la encontrada por Salvador (1994), en los universitarios valencianos, donde las actividades físicorecreativas superan porcentualmente a las actividades de competición.

Este autor expone que este avance de las actividades físico-recreativas entre el alumnado universitario es debido a su juicio a cuatro factores: la incorporación de la mujer a la vida universitaria, la falta de instalaciones en la Universidad, la falta de tiempo de los estudiantes y la curiosidad que despierta ciertas formas de hacer ejercicio (Salvador, 1994).

Es necesario, pues, como exponen Puig y Heinemann (1991; 1992), que se vayan incorporando nuevas prácticas y nuevas concepciones como complemento y alternativa al deporte colectivo tradicional, teniendo en cuenta el incremento de las ofertas de actividades físicodeportivas que se ha producido en los últimos años y que, según afirma García Ferrando (2001), probablemente continuará en el futuro, siendo muy importante adaptar esa oferta a las características y necesidades de los demandantes, aspectos que han de tener muy en cuenta los responsables de la oferta. La demanda universitaria es plural y diversificada, como afirman García Ferrando (1990) y Montiel y Sánchez (1995), y es fundamental aumentar y adaptar la oferta deportiva tradicional en la Universidad.

Otro aspecto importante que hay que señalar es el bajo porcentaje de universitarios que desean practicar actividades físico-deportivas en ofertas de la propia Universidad, quizás debido a la falta de instalaciones o la poca satisfacción de los usuarios con la calidad de las mismas (Pavón, 2004).

Los resultados del presente informe muestran claramente la tendencia hallada en las reseñadas investigaciones centradas en el alumnado almeriense, pues las actividades demandadas son principalmente las autoorganizadas, por su cuenta, de forma libre, propia del carácter individualista de los tiempo actuales, y menos competitiva, mostrando el cambio que se está produciendo en la sociedad hacia actividades que rompan con la monotonía de la vida cotidiana y urbanizada.

Estas transformaciones que está sufriendo el sistema social del deporte contemporáneo, actualmente denominado postmoderno ya lo reflejan en sus estudios autores como Puig y Heinemann (1991 y 1992), Ruiz Juan (2001) y García Ferrando (2001), los cuales hacen hincapié en la respuesta del sistema abierto que representa el deporte, ante la evolución que sufre nuestra sociedad.

Así pues, los deportes colectivos, considerados tradicionales, aunque importantes, dejan paso a otras prácticas físico-deportivas de carácter más recreativo, y desarrolladas en el medio natural. Nuestros resultados así lo reflejan, pues el porcentaje de demandantes entre la población universitaria es muy importante, llegando a obtener porcentajes importantes tanto en los fines de semana como en los periodos vacacionales de verano, Semana Santa, Navidad y puentes.

Es llamativo que entre los almerienses las modalidades físico-deportivas que se llevan a cabo en el medio natural más demandadas sean el senderismo, alpinismo, acampada, escalada, rápel, tirolina e incluso esquí alpino, coincidiendo son los estudiantes de la Universidad Politécnica de Valencia (López Yeste, 1999), en lugar de otras más relacionadas con el medio acuático, como la vela, piragüismo, remo, windsurf, etc., más propios y característicos de la costa almeriense.

Estos datos expuestos constatan la clara tendencia de los citados estudios referentes a la población universitaria almeriense, así como las investigaciones de Ispizua (1993), García Ferrando (1990; 2001), García de la Torre y Antón (1990), Salvador (1994), Tierra (1995), Herrera (1996) y López Yeste (1999), en las que se destaca la importancia de la demanda de prácticas deportivas y recreativas al aire libre y en contacto con la naturaleza, así como las relacionadas con los viajes y el turismo.

Éste es un rasgo de la sociedad postmoderna $\mathrm{y}$ que se pone de manifiesto, como expone Salvador (1994), sobre todo a partir de los años noventa. 


\section{5.- CONCLUSIONES.}

Entre las conclusiones hay que resaltar que la mayoría de los universitarios durante los días laborables desearían realizar actividades físicodeportivas cuya finalidad es el mantenimiento y mejora de la salud, en fines de semana, son los deportes tanto colectivos como individuales los más demandados, siendo esta última modalidad la más solicitada también en los periodos vacacionales de verano, Semana Santa, Navidad y puentes. También hay que señalar, la importancia que cobran las actividades físicodeportivas que se desarrollan en el medio natural en los periodos vacacionales.

Los deportes colectivos más solicitados son el fútbol, baloncesto, voleibol y fútbol-sala, mientras que entre los individuales los más señalados son la natación, tenis y ciclismo. Las actividades físico-deportivas más demandadas son el aeróbic, la carrera continua y la musculación. Por último, el senderismo, alpinismo y acampada, escalada, rapel y tirolina, esquí alpino, submarinismo y piragüismo son las actividades físico-deportivas que se practican en el medio natural más pedidas.

Finalizar señalando que la mayoría del alumnado preferiría realizar la práctica físicodeportiva por cuenta propia, de forma libre y de manera autoorganizada, mientras que el carácter o la finalidad más deseada es la no competitiva.

Por lo tanto ha quedado demostrada la importancia que tiene analizar la demanda existente en el colectivo universitario, ya que solo conociendo sus preferencias, podremos mejorar la oferta existente e influir positivamente en un estilo de vida saludable.

\section{6.- REFERENCIAS BIBLIOGRÁFICAS.}

Cañellas, A. \& Rovira, J. (1995). Los hábitos deportivos de la población adulta barcelonesa (15 a 59 años). Apunts, Educación Física y Deportes, 42, 75-79.

Consejo Superior de Deportes (2000). El Deporte español ante el siglo XXI. Madrid: Ministerio de Educación y Cultura. Autor.

García de la Torre, F., \& Antón, P. (1990). Motivaciones para la práctica físico-deportiva entre los alumnos del Campus Universitario de Álava. Apunts, Educación Física y Deportes, 22, 71-88.

García Ferrando, M. (1990). Aspectos sociales del deporte. Una reflexión sociológica. Madrid: Alianza Editorial.
García Ferrando, M. (1993). Tendencias de las prácticas físico-deportivas del alumnado universitario. En Servei d'Activitat Física de la Universitat Autònoma de Barcelona (Ed.), III Jornadas de Actividad Física y Universidad (pp. 21-38). Barcelona: Servei d'Activitat Física de la Universitat Autònoma de Barcelona.

García Ferrando, M. (2001). Los españoles y el deporte: prácticas y comportamientos en la última década del siglo XX. Encuesta sobre los hábitos deportivos de los españoles, 2000. Madrid: Ministerio de Educación, Cultura y Deporte. Consejo Superior de Deportes.

Heinemann, K. (1994). El deporte como consumo. Apunts, Educación Física y Deportes 37, 49-56.

Hernández Rodríguez, A. I. (2001). Análisis de la demanda de la comunidad universitaria almeriense en actividades físico-deportivas. Estudio de adecuación de la oferta. Tesis Doctoral, Universidad de Almería, Almería.

Hernández Rodríguez, A. I., García Montes, M. E., y Oña Sicilia, A. (2002). Las demandas de actividad físico-deportiva de los jóvenes universitarios almerienses. Motricidad. Revista de la Asociación Española de Ciencias del deporte, 8, 111-139.

Herrera, M. (1996). El control de la información como avance de gestión. En Servicio de Deportes de la Universidad de Jaén (Ed.), VI Jornadas de Deporte Universitario del Grupo Sur. Jaén: Servicio de Deportes de la Universidad de Jaén.

Ispizua, M. (1993). Hábitos deportivos de la población de la margen izquierda. Barakaldo, Portugalete, Santurtzi y Sestao. (1992). Bilbao: Ayuntamientos de Barakaldo, Portugalete, Santurtzi y Sestao. Eusko Jaurlaritza. Gobierno Vasco. Birkaiko Foru Aldundia. Diputación Foral de Bizkaia. EUDEL y KAIT.

Lizalde, E. (1993). Objetivos y programas de los servicios de actividad física. En Servei d'Activitat Física de la Universitat Autònoma de Barcelona (Ed.), III Jornadas de Actividad Física y Universidad (pp. 55-67). Barcelona: Servei d'Activitat Física de la Universitat Autònoma de Barcelona.

López Yeste, A. (1999). El deporte de la Universidad Politécnica de Valencia. Un estudio desde la psicología social del consumidor. Tesis Doctoral, Universidad de Valencia, Valencia.

Montiel, P. \& Sánchez, H. (1995). Las estrategias de promoción en los programas de actividades físico-deportivas. En Servicio de Deportes de la Universidad de Cádiz (Ed.), $5^{a s}$ Jornadas de Deporte Universitario del Grupo Sur (pp. 67-76). Cádiz: Servicio de Deportes de la Universidad de Cádiz. 
Pavón, A. (2004). Motivaciones e intereses de los universitarios murcianos hacia la práctica físicodeportiva. Tesis Doctoral, Universidad de Murcia, Murcia.

Puig, N. \& Heinemann, K. (1991). El deporte en la perspectiva del año 2000. Papers. Revista de Sociología, 38, 123-142.

Puig, N. \& Heinemann, K. (1992). El deporte en la perspectiva del año 2000. En Unisport (Ed.), El hecho deportivo: aspectos sociológicos, culturales y económicos (pp. 53-113). Málaga: Unisport. Serie apuntes 256.

Rodríguez Romo, G., Mayorga García, J. I., Merino Jiménez, Á., Garrido Muñoz, M. \& Fernández del Valle, M. (2006). La práctica, el abandono y la demanda futura de actividad física y/o deporte entre los habitantes de la Comunidad de Madrid. Kronos: la revista científica de actividad física y deporte, 9, 54-66.

Ruiz Juan, F. (2001). Análisis diferencial de los comportamientos, motivaciones y demanda de actividades físico-deportivas del alumnado almeriense de Enseñanza Secundaria Postobligatoria y de la Universidad de Almería. Tesis Doctoral, Universidad de Almería, Almería.

Ruiz Juan, F. \& García Montes, M. E. (2002). Estudio comparativo de la demanda de actividades físicodeportivas de tiempo libre, atendiendo a la frecuencia de realización, en el alumnado de Enseñanza Secundaria Posobligatoria y de Segundo Ciclo de la Universidad de Almería. Kronos: la revista científica de actividad física y deporte, 2, 45-58.
Ruiz Juan, F. \& García Montes, M. E. (2004). Demanda de actividades físico-deportivas de tiempo libre. Estudio comparativo entre el alumnado de Enseñanza Secundaria Postobligatoria y de segundo ciclo de la Universidad de Almería. Apunts, Educación Física y Deportes, 77, 63-70.

Ruiz Juan, F. \& García Montes, M. E. (2005). Hábitos físico-deportivos de los almerienses en su tiempo libre. Almería: Servicio de Publicaciones. Universidad de Almería.

Salvador, F. (1994). Una aproximación a la realidad de las actividades físico-deportivas en la Universitat de Valencia. En Servicio de Educación Física y Deportes de la Universidad de Córdoba (Ed.), IV Jornadas de Deporte Universitario del Grupo Sur, (pp. 115-121). Córdoba: Servicio de Educación Física y Deportes de la Universidad de Córdoba.

Tierra, J. (1995). Las actividades físico-deportivas y de ocio en las Universidades Andaluzas. En Servicio de Deportes de la Universidad de Cádiz (Ed.), $5^{a s}$ Jornadas de Deporte Universitario del Grupo Sur (pp. 45-65). Cádiz: Servicio de Deportes de la Universidad de Cádiz.

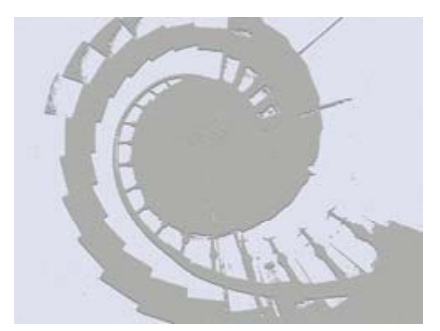

